\title{
New Curiosity Bivariate Quadratic Quaternionic Polynomials and Their Roots
}

\author{
Ilker Akkus and Gonca Kizilaslan
}

\begin{abstract}
We consider the second-order linear homogeneous quaternion recurrence solutions for some new curiosity bivariate quadratic quaternionic equations.
\end{abstract}

\section{Introduction}

The introduction section can actually be dealt with in three points of view: real, quaternion and mixed.

Real Point of View and Initial Motivation:

The integer pairs of solutions of the quadratic equations $a x^{2}+b x y+c y^{2}+$ $d=0$ for some special integral numbers $a, b, c$, and $d$ are found interesting by several researchers, see $[3,4]$. The well-known general form of a real quadratic form is as follows:

A real standard quadratic form $q$ in $n$-variables $x_{i}, 1 \leq i \leq n$, over a $K$ field is a homogeneous polynomial structure such that every term has degree two with coefficients in $K$; i.e,

$$
q\left(x_{1}, x_{2}, \ldots, x_{n}\right)=\sum_{i} c_{i} x_{i}^{2}+\sum_{i<j} d_{i j} x_{i} x_{j} .
$$

Key Words: Quaternion, Real quadratic form, Quadratic quaternionic equation, Polynomials with mixed quaternion coefficients.

2010 Mathematics Subject Classification: Primary 11R52, 11D09; Secondary 15A63.

Received: 01.03.2020

Accepted: 01.05.2020 
If it is defined on the field $K$, let us emphasize that our only constraint is that the characteristic of $K$ must be different from 2. Some types of quadratic forms are particularly concerned with conical sections. Hence, the conical sections are pleased by successive pairs terms of the well-known Lucas and Fibonacci integer number sequences are also found considering by many authors, see $[9,10]$.

We will give a brief introduction to quaternion algebra and polynomials over quaternion algebra, as we will deal with quadratic quaternionic equations.

Fundamentals of Quaternion Algebras:

Definition 1.1. The Hamilton's quaternions are defined by

$$
\mathbf{H}:=\left\{a_{0}+a_{1} \mathbf{i}+a_{2} \mathbf{j}+a_{3} \mathbf{k} \mid a_{i} \in \mathbb{R}, 0 \leq i \leq 3\right\}
$$

where

$$
\mathbf{i}^{2}=\mathbf{j}^{2}=\mathbf{k}^{2}=\mathbf{i j k}=-1 .
$$

For a quaternion $q=a_{0}+a_{1} \mathbf{i}+a_{2} \mathbf{j}+a_{3} \mathbf{k}$, its conjugate $q^{*}$ is the quaternion $q^{*}=a_{0}-a_{1} \mathbf{i}+a_{2} \mathbf{j}+a_{3} \mathbf{k}$. The norm and trace of the quaternion $q$ are denoted by $N(q)$ and $\operatorname{Tr}(q)$, respectively and defined as

$$
\operatorname{Tr}(q)=2 a_{0} \text { and } N(q)=a_{0}^{2}+a_{1}^{2}+a_{2}^{2}+a_{3}^{2} .
$$

Second-Order Linear Quaternionic Recurrence Relations:

Many researchers studied the quaternions which are called as quaternion sequences by taking components from different well-known special number sequences. One of the earliest studies are about the quaternionic homogeneous recurrence sequences with components Lucas and Fibonacci numbers. In [7], Horadam described the second-order homogeneous Lucas quaternion sequence and Fibonacci quaternion sequence as

$$
V_{n}=L_{n}+L_{n+1} \mathbf{i}+L_{n+2} \mathbf{j}+L_{n+3} \mathbf{k}
$$

and

$$
Q_{n}=F_{n}+F_{n+1} \mathbf{i}+F_{n+2} \mathbf{j}+F_{n+3} \mathbf{k}
$$

where $L_{n}$ and $F_{n}$ are the $n t h$ Lucas numbers and $n t h$ Fibonacci number, respectively. In [8], a generalization of Fibonacci quaternion sequence is studied.

Definition $1.2([8])$. Let $n \geq 0$ be an integer and $q$ and $p$ be nonzero real numbers such that $p^{2}+4 q>0 .(p, q)-$ Fibonacci sequence $\mathcal{F}_{n}:=\mathcal{F}_{n}(p, q)$ is described by the following recursive relation:

$$
\mathcal{F}_{n}=q \mathcal{F}_{n-2}+p \mathcal{F}_{n-1}
$$


with $\mathcal{F}_{0}=0$ and $\mathcal{F}_{1}=1$. Then using this number sequence, $(p, q)-$ Fibonacci quaternion sequence $\mathcal{Q F}_{n}:=\mathcal{Q F}_{n}(p, q)$ is described by the following quaternionic recursive relation:

$$
2 \mathcal{F}_{n-3}=\mathcal{F}_{n-3}+\mathcal{F}_{n-2} \mathbf{i}+\mathcal{F}_{n-1} \mathbf{j}+\mathcal{F}_{n} \mathbf{k} .
$$

Quaternion Point of View:

A monic quaternion polynomial structure with $n$ degree in the quaternion indeterminate $x$ is defined by

$$
p(x)=x^{n}+q_{n-1} x^{n-1}+\cdots+q_{0}
$$

where $q_{0}, q_{1}, \cdots, q_{n-1}$ are quaternions. We call that $\alpha$ is a root of the quaternionic polynomial structure $p(x)$ if $p(\alpha)=0$. The roots of polynomials over special algebraic structures are considered by some researchers, see $[2,6,11$, 12]. Niven proves the existence of a quaternion root of polynomials over real quaternions with a simple algorithm which based on the norm and trace of a quaternion [11]. In [14], the authors give a more practical perspective to find the norm and trace by using the fact that the norm and the trace of the zero can be found by the companion matrix related to the polynomial. In [12], another algorithm is given to find the roots of a polynomial over quaternion algebra.

In [16], authors are interested in the equations of the following form

$$
x^{2}+\sum_{j=1}^{t} \alpha^{(j)} x \beta^{(j)}+\sum_{j=1}^{s} \gamma^{(j)} x^{*} \delta^{(j)}=-d
$$

where

$$
\begin{aligned}
\alpha^{(j)} & =\alpha_{0}^{(j)}+\alpha_{1}^{(j)} \mathbf{i}+\alpha_{2}^{(j)} \mathbf{j}+\alpha_{3}^{(j)} \mathbf{k}, \\
\beta^{(j)} & =\beta_{0}^{(j)}+\beta_{1}^{(j)} \mathbf{i}+\beta_{2}^{(j)} \mathbf{j}+\beta_{3}^{(j)} \mathbf{k}, \\
\gamma^{(i)} & =\gamma_{0}^{(i)}+\gamma_{1}^{(i)} \mathbf{i}+\gamma_{2}^{(i)} \mathbf{j}+\gamma_{3}^{(i)} \mathbf{k}, \\
\delta^{(j)} & =\delta_{0}^{(i)}+\delta_{1}^{(i)} \mathbf{i}+\delta_{2}^{(i)} \mathbf{j}+\delta_{3}^{(i)} \mathbf{k}, \\
d & =d_{0}+d_{1} \mathbf{i}+d_{2} \mathbf{j}+d_{3} \mathbf{k}
\end{aligned}
$$

are quaternions and $\alpha_{m}^{(j)}, \beta_{m}^{(j)}, \gamma_{m}^{(i)}, \delta_{m}^{(i)}, d_{m}$ are real numbers, $m=0,1,2,3$, $j=1,2, \cdots, t, i=1,2, \cdots, s$ and $t, s$ are nonnegative integers. For any quaternion $q=q_{0}+q_{1} \mathbf{i}+q_{2} \mathbf{j}+q_{3} \mathbf{k}$, they defined the matrices $R(q), I(q), J(q)$ and $K(q)$, see [16], in the following way:

$$
R(q)=\left[\begin{array}{cccc}
q_{0} & -q_{1} & -q_{2} & -q_{3} \\
-q_{1} & -q_{0} & q_{3} & -q_{2} \\
-q_{2} & -q_{3} & -q_{0} & q_{1} \\
-q_{3} & q_{2} & -q_{1} & -q_{0}
\end{array}\right], I(q)=\left[\begin{array}{cccc}
q_{1} & q_{0} & q_{3} & -q_{2} \\
q_{0} & -q_{1} & q_{2} & q_{3} \\
q_{3} & -q_{2} & -q_{1} & -q_{0} \\
-q_{2} & -q_{3} & q_{0} & -q_{1}
\end{array}\right],
$$




$$
J(q)=\left[\begin{array}{cccc}
q_{2} & -q_{3} & q_{0} & q_{1} \\
-q_{3} & -q_{2} & -q_{1} & q_{0} \\
q_{0} & q_{1} & -q_{2} & q_{3} \\
q_{1} & -q_{0} & -q_{3} & -q_{2}
\end{array}\right], K(q)=\left[\begin{array}{cccc}
q_{3} & q_{2} & -q_{1} & q_{0} \\
q_{2} & -q_{3} & -q_{0} & -q_{1} \\
-q_{1} & q_{0} & -q_{3} & -q_{2} \\
q_{0} & q_{1} & q_{2} & -q_{3}
\end{array}\right]
$$

and obtained the following result.

Theorem 1.3 ([16], Theorem 2.1.). $x=a_{0}+a_{1} \mathbf{i}+a_{2} \mathbf{j}+a_{3} \mathbf{k}$ is a solution of (1.2) if and only if $\left(a_{0}, a_{1}, a_{2}, a_{3}\right)$ fulfills the equations

$$
g_{l}\left(a_{0}, a_{1}, a_{2}, a_{3}\right)=0, \quad l=0, \cdots, 3 .
$$

Here

$$
\begin{aligned}
& g_{0}=a_{0}^{2}-a_{1}^{2}-a_{2}^{2}-a_{3}^{2}+ \\
& \left(a_{0}, a_{1}, a_{2}, a_{3}\right)\left\{\sum_{j=1}^{t} R\left(\beta^{(j)}\right)\left[\begin{array}{l}
\alpha_{0}^{(j)} \\
\alpha_{1}^{(j)} \\
\alpha_{2}^{(j)} \\
\alpha_{3}^{(j)}
\end{array}\right]+\sum_{i=1}^{s} D R\left(\delta^{(i)}\right)\left[\begin{array}{c}
\gamma_{0}^{(i)} \\
\gamma_{1}^{(i)} \\
\gamma_{2}^{(i)} \\
\gamma_{3}^{(i)}
\end{array}\right]\right\}+d_{0}, \\
& g_{1}=2 a_{0} a_{1}+ \\
& \left(a_{0}, a_{1}, a_{2}, a_{3}\right)\left\{\sum_{j=1}^{t} I\left(\beta^{(j)}\right)\left[\begin{array}{l}
\alpha_{0}^{(j)} \\
\alpha_{1}^{(j)} \\
\alpha_{2}^{(j)} \\
\alpha_{3}^{(j)}
\end{array}\right]+\sum_{i=1}^{s} D I\left(\delta^{(i)}\right)\left[\begin{array}{c}
\gamma_{0}^{(i)} \\
\gamma_{1}^{(i)} \\
\gamma_{2}^{(i)} \\
\gamma_{3}^{(i)}
\end{array}\right]\right\}+d_{1}, \\
& g_{2}=2 a_{0} a_{2}+ \\
& \left(a_{0}, a_{1}, a_{2}, a_{3}\right)\left\{\sum_{j=1}^{t} J\left(\beta^{(j)}\right)\left[\begin{array}{l}
\alpha_{0}^{(j)} \\
\alpha_{1}^{(j)} \\
\alpha_{2}^{(j)} \\
\alpha_{3}^{(j)}
\end{array}\right]+\sum_{i=1}^{s} D J\left(\delta^{(i)}\right)\left[\begin{array}{c}
\gamma_{0}^{(i)} \\
\gamma_{1}^{(i)} \\
\gamma_{2}^{(i)} \\
\gamma_{3}^{(i)}
\end{array}\right]\right\}+d_{2}, \\
& g_{3}=2 a_{0} a_{3}+ \\
& \left(a_{0}, a_{1}, a_{2}, a_{3}\right)\left\{\sum_{j=1}^{t} K\left(\beta^{(j)}\right)\left[\begin{array}{l}
\alpha_{0}^{(j)} \\
\alpha_{1}^{(j)} \\
\alpha_{2}^{(j)} \\
\alpha_{3}^{(j)}
\end{array}\right]+\sum_{i=1}^{s} D K\left(\delta^{(i)}\right)\left[\begin{array}{c}
\gamma_{0}^{(i)} \\
\gamma_{1}^{(i)} \\
\gamma_{2}^{(i)} \\
\gamma_{3}^{(i)}
\end{array}\right]\right\}+d_{3}
\end{aligned}
$$

and $D=\operatorname{diag}(1,-1,-1,-1)$.

Quadratic left polynomials are also studied in [13]. These polynomials are considered over the ring of split quaternions. They are interested in factorizability of a quadratic split quaternion polynomial. 
The linear quaternionic equations are also found interesting. In [15], the solutions of the linear quaternion equation are considered. In [5], they study some equations in generalized octonion and quaternion algebras.

Mixed Point of View:

As the equations which are satisfied by the pairs of Lucas and Fibonacci numbers are found interesting by many authors, we think that quaternionic equations whose solutions are pairs of Lucas and Fibonacci quaternions will also be interesting. In [1], we are interested in some quaternionic equations and found the Lucas and Fibonacci quaternion pairs of solutions. In the current paper, we consider the quaternion zeros with integer components of some equations of the following form

$$
x^{2}-a x y \pm y^{2}=\tilde{q},
$$

where $x, y$ and $\tilde{q}$ are quaternions and $a$ is an integer. Also, after some experiments, we obtain that for $m \geq 2$, the curiosity generalized equation

$x^{2}-\left(2^{2 m}-2\right) x y+y^{2}-2^{m+1}\left[-2^{m}+\mathbf{i}+\left(2^{3 m}-2^{m+1}\right) \mathbf{j}+\left(1-2^{2 m+1}+2^{4 m-1}\right) \mathbf{k}\right]=0$

has $(p, q)$-Fibonacci quaternion solutions according to the selection of the quaternion $y$.

\section{Solutions of Some Bivariate Quadratic Quaternionic Equations}

Throughout this section, let $m$ be an integer and $x=x_{0}+x_{1} \mathbf{i}+x_{2} \mathbf{j}+x_{3} \mathbf{k}$ be a quaternion with integer coefficients.

\subsection{Some Bivariate Quadratic Quaternionic Equations and Their Commutative Correspondences}

Definition 2.1. Let $a, b, c$ be reals and $q_{1}$ and $q_{2}$ are real quaternions. The quadratic quaternionic equations $a y^{2}+b x y+c x^{2}+q_{1}=0$ and $a y^{2}+b y x+$ $c x^{2}+q_{2}=0$ are called commutative correspondences when they have a same quaternion solution with integer coefficients.

The following results can be obtained easily by Definition 2.1.

Proposition 2.2. If the quadratic quaternionic equations $f(x, y):=a y^{2}+$ $b x y+c x^{2}+q_{1}=0$ and $g(x, y):=a y^{2}+b y x+c x^{2}+q_{2}=0$ are commutative correspondences, then the equation $m f(x, y)+n g(x, y)=0$ for real numbers $m$ and $n$ becomes also commutative correspondences with them. 
Proposition 2.3. Commutative correspondency is an equivalence relation.

We will consider the bivariate quadratic quaternionic equations in the following forms and their some commutative correspondences

$$
x^{2}-x y-y^{2}+q_{1}=0 \text { and } x^{2}-3 x y+y^{2}+q_{2}=0,
$$

where $q_{1}, q_{2}$ are some real quaternions. In these types we will find the solutions by using their equivalent real quadratic forms.

Theorem 2.4. Let quaternion y be $m$ times the Lucas quaternion. Then the equations

$$
x^{2}-x y-y^{2}+5 m^{2}(2+2 \mathbf{j}+5 \mathbf{k})=0
$$

and

$$
\left.x^{2}-y x-y^{2}+10 m^{2}+10 m^{2} \mathbf{i}+20 m^{2} \mathbf{j}+15 m^{2} \mathbf{k}\right)=0
$$

are commutative correspondences. The quaternion solutions whose coefficients are integers are of the form $(x, y)= \pm\left(m V_{2 n+1}, m V_{2 n}\right)$.

Proof. We will find the quaternion zeros with integer components of the above equation (2.1). Equation (2.2) can be solved similarly.

Let $y=m V_{2 n}$. It can be shown that

$$
\begin{aligned}
V_{2 n}^{2}= & -N\left(V_{2 n}\right)+2 L_{2 n}^{2}+2 L_{2 n} L_{2 n+1} \mathbf{i}+2 L_{2 n} L_{2 n+2} \mathbf{j}+2 L_{2 n} L_{2 n+3} \mathbf{k} \\
= & -15 F_{4 n+3}+2 L_{2 n}^{2}+2 L_{2 n} L_{2 n+1} \mathbf{i}+ \\
& 2 L_{2 n} L_{2 n+2} \mathbf{j}+2 L_{2 n} L_{2 n+3} \mathbf{k} .
\end{aligned}
$$

Then we have

$$
\begin{aligned}
x_{0}^{2}-x_{1}^{2}-x_{2}^{2}-x_{3}^{2}-m\left(L_{2 n} x_{0}-L_{2 n+1} x_{1}-L_{2 n+2} x_{2}-L_{2 n+3} x_{3}\right)+d_{0}=0 \\
2 x_{0} x_{1}-m\left(L_{2 n+1} x_{0}+L_{2 n} x_{1}+L_{2 n+3} x_{2}-L_{2 n+2} x_{3}\right)+d_{1}=0 \\
2 x_{0} x_{2}-m\left(L_{2 n+2} x_{0}-L_{2 n+3} x_{1}+L_{2 n} x_{2}+L_{2 n+1} x_{3}\right)+d_{2}=0 \\
2 x_{0} x_{3}-m\left(L_{2 n+3} x_{0}+L_{2 n+2} x_{1}-L_{2 n+1} x_{2}+L_{2 n} x_{3}\right)+d_{3}=0
\end{aligned}
$$

where

$$
\begin{aligned}
d= & -m^{2} V_{2 n}^{2}+5 m^{2}(2+2 \mathbf{j}+5 \mathbf{k}) \\
= & m^{2}\left[10+15 F_{4 n+3}-2 L_{2 n}^{2}-2 L_{2 n} L_{2 n+1} \mathbf{i}+\left(10-2 L_{2 n} L_{2 n+2}\right) \mathbf{j}+\right. \\
& \left.\left(25-2 L_{2 n} L_{2 n+3}\right) \mathbf{k}\right] \\
= & d_{0}+d_{1} \mathbf{i}+d_{2} \mathbf{j}+d_{3} \mathbf{k} .
\end{aligned}
$$

From these equations we obtain the zero $x=m V_{2 n+1}$. A similar consideration shows that when $y=-m V_{2 n}$, we have the zero $x=-m V_{2 n+1}$. 
Let $y=m V_{2 n+1}$. Since

$$
\begin{aligned}
V_{2 n+1}^{2}= & -N\left(V_{2 n+1}\right)+2 L_{2 n+1}^{2}+2 L_{2 n+1} L_{2 n+2} \mathbf{i}+ \\
& 2 L_{2 n+1} L_{2 n+3} \mathbf{j}+2 L_{2 n+1} L_{2 n+4} \mathbf{k} \\
= & -15 F_{4 n+5}+2 L_{2 n+1}^{2}+2 L_{2 n+1} L_{2 n+2} \mathbf{i}+ \\
& 2 L_{2 n+1} L_{2 n+3} \mathbf{j}+2 L_{2 n+1} L_{2 n+4} \mathbf{k},
\end{aligned}
$$

we have the following real quadratic form

$$
\begin{aligned}
x_{0}^{2}- & x_{1}^{2}-x_{2}^{2}-x_{3}^{2}-m\left(L_{2 n+1} x_{0}-L_{2 n+2} x_{1}-L_{2 n+3} x_{2}-L_{2 n+4} x_{3}\right)+d_{0}=0 \\
& 2 x_{0} x_{1}-m\left(L_{2 n+2} x_{0}+L_{2 n+1} x_{1}+L_{2 n+4} x_{2}-L_{2 n+3} x_{3}\right)+d_{1}=0 \\
& 2 x_{0} x_{2}-m\left(L_{2 n+3} x_{0}-L_{2 n+4} x_{1}+L_{2 n+1} x_{2}+L_{2 n+2} x_{3}\right)+d_{2}=0 \\
& 2 x_{0} x_{3}-m\left(L_{2 n+4} x_{0}+L_{2 n+3} x_{1}-L_{2 n+2} x_{2}+L_{2 n+1} x_{3}\right)+d_{3}=0
\end{aligned}
$$

where

$$
\begin{aligned}
d= & -m^{2} V_{2 n+1}^{2}+5 m^{2}(2+2 \mathbf{j}+5 \mathbf{k}) \\
= & m^{2}\left[10+15 F_{4 n+5}-2 L_{2 n+1}^{2}-2 L_{2 n+1} L_{2 n+2} \mathbf{i}+\left(10-2 L_{2 n+1} L_{2 n+3}\right) \mathbf{j}+\right. \\
& \left.\left(25-2 L_{2 n+1} L_{2 n+4}\right) \mathbf{k}\right] \\
= & d_{0}+d_{1} \mathbf{i}+d_{2} \mathbf{j}+d_{3} \mathbf{k} .
\end{aligned}
$$

But this form does not have real solutions. Similarly for $y=-m V_{2 n+1}$, the real quadratic form has no solutions.

Theorem 2.5. Let quaternion y be $m$ times the Lucas quaternion. Then the equations

$$
x^{2}-3 x y+y^{2}+5 m^{2}(2-2 \mathbf{i}+7 \mathbf{k})=0
$$

and

$$
x^{2}-3 y x+y^{2}+5 m^{2}(2+4 \mathbf{i}+6 \mathbf{j}+\mathbf{k})=0
$$

are commutative correspondences. The quaternion solutions whose coefficients are integers are given by $(x, y)= \pm\left(m V_{2 n+2}, m V_{2 n}\right)$.

Proof. We will find the quaternion zeros with integer components of the above equation (2.5). Equation (2.6) can be solved similarly. tions

Let $y=m V_{2 n}$. Then using Equation (2.3) we obtain the following equa-

$$
\begin{aligned}
x_{0}^{2}- & x_{1}^{2}-x_{2}^{2}-x_{3}^{2}-3 m\left(L_{2 n} x_{0}-L_{2 n+1} x_{1}-L_{2 n+2} x_{2}-L_{2 n+3} x_{3}\right)+d_{0}=0 \\
& 2 x_{0} x_{1}-3 m\left(L_{2 n+1} x_{0}+L_{2 n} x_{1}+L_{2 n+3} x_{2}-L_{2 n+2} x_{3}\right)+d_{1}=0 \\
& 2 x_{0} x_{2}-3 m\left(L_{2 n+2} x_{0}-L_{2 n+3} x_{1}+L_{2 n} x_{2}+L_{2 n+1} x_{3}\right)+d_{2}=0
\end{aligned}
$$




$$
2 x_{0} x_{3}-3 m\left(L_{2 n+3} x_{0}+L_{2 n+2} x_{1}-L_{2 n+1} x_{2}+L_{2 n} x_{3}\right)+d_{3}=0
$$

where

$$
\begin{aligned}
d= & m^{2} V_{2 n+1}^{2}+5 m^{2}(2-2 \mathbf{i}+7 \mathbf{k}) \\
= & m^{2}\left[10-15 F_{4 n+5}+2 L_{2 n}^{2}+\left(2 L_{2 n} L_{2 n+1}-10\right) \mathbf{i}+2 L_{2 n} L_{2 n+2} \mathbf{j}+\right. \\
& \left.\left(35+2 L_{2 n} L_{2 n+3}\right) \mathbf{k}\right] \\
= & d_{0}+d_{1} \mathbf{i}+d_{2} \mathbf{j}+d_{3} \mathbf{k}
\end{aligned}
$$

From these equations we obtain the zero $x=m V_{2 n+2}$. A similar consideration shows that when $y=-m V_{2 n}$, we have the zero $x=-m V_{2 n+2}$.

Let $y=m V_{2 n+1}$. Using Equation (2.4), the real quadratic form is

$$
\begin{aligned}
x_{0}^{2}-x_{1}^{2}-x_{2}^{2}-x_{3}^{2}-3 m\left(L_{2 n+1} x_{0}-L_{2 n+2} x_{1}-L_{2 n+3} x_{2}-L_{2 n+4} x_{3}\right)+d_{0}=0 \\
2 x_{0} x_{1}-3 m\left(L_{2 n+2} x_{0}+L_{2 n+1} x_{1}+L_{2 n+4} x_{2}-L_{2 n+3} x_{3}\right)+d_{1}=0 \\
2 x_{0} x_{2}-3 m\left(L_{2 n+3} x_{0}-L_{2 n+4} x_{1}+L_{2 n+1} x_{2}+L_{2 n+2} x_{3}\right)+d_{2}=0 \\
2 x_{0} x_{3}-3 m\left(L_{2 n+4} x_{0}+L_{2 n+3} x_{1}-L_{2 n+2} x_{2}+L_{2 n+1} x_{3}\right)+d_{3}=0
\end{aligned}
$$

where

$$
\begin{aligned}
d= & m^{2} V_{2 n+1}^{2}+5 m^{2}(2-2 \mathbf{i}+7 \mathbf{k}) \\
= & m^{2}\left[10-15 F_{4 n+5}+2 L_{2 n+1}^{2}+\left(2 L_{2 n+1} L_{2 n+2}-10\right) \mathbf{i}+2 L_{2 n+1} L_{2 n+3} \mathbf{j}+\right. \\
& \left.\left(35+2 L_{2 n+1} L_{2 n+4}\right) \mathbf{k}\right] \\
= & d_{0}+d_{1} \mathbf{i}+d_{2} \mathbf{j}+d_{3} \mathbf{k} .
\end{aligned}
$$

But this form does not have real solutions. Similarly for $y=-m V_{2 n+1}$, the real quadratic form has no solutions.

\subsection{A Curiosity Generalized Bivariate Quadratic Quaternionic Equa- tion}

Now, we will consider the quaternion zeros of some special quaternionic polynomials and then obtain a general polynomial with generalized Fibonacci quaternion zeros.

Theorem 2.6. Let $y$ be the generalized Fibonacci quaternion $Q_{\mathcal{F}} \mathcal{F}_{n-1}(4,-1)$. The equation

$$
x^{2}-14 x y+y^{2}+32-8 \mathbf{i}-448 \mathbf{j}-776 \mathbf{k}=0
$$

has the quaternion solutions whose coefficients are integers in the form $(x, y)=\left(\mathcal{Q F}_{n+1}(4,-1), \mathcal{Q F}_{n-1}(4,-1)\right)$. 
Proof. Let $y=Q \mathcal{F}_{n-1}(4,-1)$. Let us denote $\mathcal{F}_{n}(4,-1)$ simply by $U_{n}$. It can be easily shown that

$2 \mathcal{F}_{n-1}^{2}(4,-1)=14 U_{n}^{2}-16 U_{n+1}^{2}+2 U_{n-1} U_{n} \mathbf{i}+2 U_{n-1} U_{n+1} \mathbf{j}+2 U_{n-1} U_{n+2} \mathbf{k}$.

Then the equivalent real quadratic form will be

$$
\begin{aligned}
x_{0}^{2}-x_{1}^{2}-x_{2}^{2}-x_{3}^{2}-14\left(U_{n-1} x_{0}-U_{n} x_{1}-U_{n+1} x_{2}-U_{n+2} x_{3}\right)+d_{0}=0 \\
2 x_{0} x_{1}-14\left(U_{n} x_{0}+U_{n-1} x_{1}+U_{n+2} x_{2}-U_{n+1} x_{3}\right)+d_{1}=0 \\
2 x_{0} x_{2}-14\left(U_{n+1} x_{0}-U_{n+2} x_{1}+U_{n-1} x_{2}+U_{n} x_{3}\right)+d_{2}=0 \\
2 x_{0} x_{3}-14\left(U_{n+2} x_{0}+U_{n+1} x_{1}-U_{n} x_{2}-U_{n-1} x_{3}\right)+d_{3}=0
\end{aligned}
$$

where

$$
\begin{aligned}
d= & 2 \mathcal{F}_{n-1}^{2}(4,-1)-8(-4+\mathbf{i}+56 \mathbf{j}+97 \mathbf{k}) \\
= & 32+14 U_{n}^{2}-16 U_{n+1}^{2}+\left(2 U_{n-1} U_{n}-8\right) \mathbf{i}+\left(2 U_{n-1} U_{n+1}-448\right) \mathbf{j}+ \\
& \left(2 U_{n-1} U_{n+2}-776\right) \mathbf{k} \\
= & d_{0}+d_{1} \mathbf{i}+d_{2} \mathbf{j}+d_{3} \mathbf{k} .
\end{aligned}
$$

From these equations we obtain that $x=\mathcal{Q F}_{n+1}(4,-1)$.

Theorem 2.7. Let $y$ be the generalized Fibonacci quaternion $2 \mathcal{F}_{n-1}(8,-1)$. The equation

$$
x^{2}-62 x y+y^{2}+128-16 \mathbf{i}-7936 \mathbf{j}-30736 \mathbf{k}=0
$$

has the quaternion solutions whose coefficients are integers in the form $(x, y)=\left(\mathcal{Q F}_{n+1}(8,-1), \mathcal{Q F}_{n-1}(8,-1)\right)$.

Proof. Let $y=2 \mathcal{F}_{n-1}(8,-1)$. Let us denote $\mathcal{F}_{n}(8,-1)$ simply by $U_{n}$. It can be easily shown that

$2 \mathcal{F}_{n-1}^{2}(8,-1)=62 U_{n}^{2}-64 U_{n+1}^{2}+2 U_{n-1} U_{n} \mathbf{i}+2 U_{n-1} U_{n+1} \mathbf{j}+2 U_{n-1} U_{n+2} \mathbf{k}$.

Then the equivalent real quadratic form will be

$$
\begin{aligned}
x_{0}^{2}- & x_{1}^{2}-x_{2}^{2}-x_{3}^{2}-62\left(U_{n-1} x_{0}-U_{n} x_{1}-U_{n+1} x_{2}-U_{n+2} x_{3}\right)+d_{0}=0 \\
& 2 x_{0} x_{1}-62\left(U_{n} x_{0}+U_{n-1} x_{1}+U_{n+2} x_{2}-U_{n+1} x_{3}\right)+d_{1}=0 \\
& 2 x_{0} x_{2}-62\left(U_{n+1} x_{0}-U_{n+2} x_{1}+U_{n-1} x_{2}+U_{n} x_{3}\right)+d_{2}=0 \\
& 2 x_{0} x_{3}-62\left(U_{n+2} x_{0}+U_{n+1} x_{1}-U_{n} x_{2}-U_{n-1} x_{3}\right)+d_{3}=0
\end{aligned}
$$

where

$$
d=\mathcal{Q F}_{n-1}^{2}(8,-1)-16(-8+\mathbf{i}+496 \mathbf{j}+1921 \mathbf{k})
$$




$$
\begin{aligned}
= & 128+62 U_{n}^{2}-64 U_{n+1}^{2}+\left(2 U_{n-1} U_{n}-16\right) \mathbf{i}+\left(2 U_{n-1} U_{n+1}-7936\right) \mathbf{j}+ \\
& \left(2 U_{n-1} U_{n+2}-30736\right) \mathbf{k} \\
= & d_{0}+d_{1} \mathbf{i}+d_{2} \mathbf{j}+d_{3} \mathbf{k} .
\end{aligned}
$$

From these equations we obtain that $x=2 \mathcal{F}_{n+1}(8,-1)$.

Theorem 2.8. Let $y$ be the generalized Fibonacci quaternion $2 \mathcal{F}_{n-1}(16,-1)$. Then the equation

$$
x^{2}-62 x y+y^{2}+4064-254 \mathbf{i}-1032256 \mathbf{j}-8193278 \mathbf{k}=0
$$

has the quaternion solutions whose coefficients are integers in the form $(x, y)=\left(\mathcal{Q F}_{n+1}(16,-1), \mathcal{Q F}_{n-1}(16,-1)\right)$.

Proof. Let $y=\mathcal{Q F}_{n-1}(16,-1)$. Let us denote $\mathcal{F}_{n}(16,-1)$ simply by $U_{n}$. It can be easily shown that

$\mathcal{Q F}_{n-1}^{2}(16,-1)=254 U_{n}^{2}-256 U_{n+1}^{2}+2 U_{n-1} U_{n} \mathbf{i}+2 U_{n-1} U_{n+1} \mathbf{j}+2 U_{n-1} U_{n+2} \mathbf{k}$.

Then the equivalent real quadratic form will be

$$
\begin{aligned}
x_{0}^{2}- & x_{1}^{2}-x_{2}^{2}-x_{3}^{2}-254\left(U_{n-1} x_{0}-U_{n} x_{1}-U_{n+1} x_{2}-U_{n+2} x_{3}\right)+d_{0}=0 \\
& 2 x_{0} x_{1}-254\left(U_{n} x_{0}+U_{n-1} x_{1}+U_{n+2} x_{2}-U_{n+1} x_{3}\right)+d_{1}=0 \\
& 2 x_{0} x_{2}-254\left(U_{n+1} x_{0}-U_{n+2} x_{1}+U_{n-1} x_{2}+U_{n} x_{3}\right)+d_{2}=0 \\
& 2 x_{0} x_{3}-254\left(U_{n+2} x_{0}+U_{n+1} x_{1}-U_{n} x_{2}-U_{n-1} x_{3}\right)+d_{3}=0
\end{aligned}
$$

where

$$
\begin{aligned}
d= & \mathcal{Q F}{ }_{n-1}^{2}(16,-1)-254(-16+\mathbf{i}+4064 \mathbf{j}+32257 \mathbf{k}) \\
= & 512+254 U_{n}^{2}-256 U_{n+1}^{2}+\left(2 U_{n-1} U_{n}-32\right) \mathbf{i}+ \\
& \left(2 U_{n-1} U_{n+1}-130048\right) \mathbf{j}+\left(2 U_{n-1} U_{n+2}-1032224\right) \mathbf{k} \\
= & d_{0}+d_{1} \mathbf{i}+d_{2} \mathbf{j}+d_{3} \mathbf{k} .
\end{aligned}
$$

From these equations we obtain that $x=Q^{\mathcal{F}} \mathcal{F}_{n+1}(16,-1)$.

Inspiring by the polynomials given above, the following theorem gives solutions of a generalized polynomial.

Theorem 2.9. Let $y$ be the generalized Fibonacci quaternion $Q \mathcal{F}_{n-1}\left(2^{m},-1\right)$. For $m \geq 2$, the only quaternion zeros with integer components of the following equation

$x^{2}-\left(2^{2 m}-2\right) x y+y^{2}-2^{m+1}\left[-2^{m}+\mathbf{i}+\left(2^{3 m}-2^{m+1}\right) \mathbf{j}+\left(1-2^{2 m+1}+2^{4 m-1}\right) \mathbf{k}\right]=0$

are given by $(x, y)=\left(\mathcal{Q F}_{n+1}\left(2^{m},-1\right), \mathcal{Q F}_{n-1}\left(2^{m},-1\right)\right)$. 
Proof. Let $m \geq 2$ and $y=\mathcal{Q F}_{n-1}\left(2^{m},-1\right)$. Let us denote $\mathcal{F}_{n}\left(2^{m},-1\right)$ simply by $U_{n}$. It can be easily shown that

$$
\begin{aligned}
\mathcal{Q F}_{n-1}^{2}\left(2^{m},-1\right)= & \left(2^{2 m}-2\right) U_{n}^{2}-2^{2 m} U_{n+1}^{2}+2 U_{n-1} U_{n} \mathbf{i}+ \\
& 2 U_{n-1} U_{n+1} \mathbf{j}+2 U_{n-1} U_{n+2} \mathbf{k} .
\end{aligned}
$$

Then the equivalent real quadratic form will be

$$
\begin{gathered}
x_{0}^{2}-x_{1}^{2}-x_{2}^{2}-x_{3}^{2}-\left(2^{2 m}-2\right)\left(U_{n-1} x_{0}-U_{n} x_{1}-U_{n+1} x_{2}-U_{n+2} x_{3}\right)+d_{0}=0 \\
2 x_{0} x_{1}-\left(2^{2 m}-2\right)\left(U_{n} x_{0}+U_{n-1} x_{1}+U_{n+2} x_{2}-U_{n+1} x_{3}\right)+d_{1}=0 \\
2 x_{0} x_{2}-\left(2^{2 m}-2\right)\left(U_{n+1} x_{0}-U_{n+2} x_{1}+U_{n-1} x_{2}+U_{n} x_{3}\right)+d_{2}=0 \\
2 x_{0} x_{3}-\left(2^{2 m}-2\right)\left(U_{n+2} x_{0}+U_{n+1} x_{1}-U_{n} x_{2}-U_{n-1} x_{3}\right)+d_{3}=0
\end{gathered}
$$

where

$$
\begin{aligned}
d= & 2 \mathcal{F}_{n-1}^{2}\left(2^{m},-1\right)-\left(2^{m+1}\right)\left(-2^{m}+\mathbf{i}+\left(2^{3 m}-2^{m+1}\right) \mathbf{j}+\right. \\
& \left.\left(1-2^{2 m+1}+2^{4 m-1}\right) \mathbf{k}\right) \\
= & 2^{m+2}+\left(2^{2 m}-2\right) U_{n}^{2}-2^{2 m} U_{n+1}^{2}+\left(2 U_{n-1} U_{n}-2^{4 m+1}+2^{2 m+2}\right) \mathbf{i}+ \\
& \left(2 U_{n-1} U_{n+1}-2^{m+1}+2^{3 m+2}\right) \mathbf{j}+\left(2 U_{n-1} U_{n+2}-2^{5 m}\right) \mathbf{k} \\
= & d_{0}+d_{1} \mathbf{i}+d_{2} \mathbf{j}+d_{3} \mathbf{k} .
\end{aligned}
$$

From these equations we obtain that $x=\mathcal{Q F}_{n+1}\left(2^{m},-1\right)$.

\section{References}

[1] I. Akkus, G. Kizilaslan, Some new quater. quad. with zeros in terms of sec. ord. quater. recur., Advances Appl. Clif. Algebras, 29 (1) (2019), Art. 14, 14 pp.

[2] U. Bray, G. Whaples, Polynom. with coeffic. from a div. ring, Can. J. Math., (35) (1983), 509-515.

[3] J. H. E. Cohn, The Diop. equat. $x^{4}-D y^{2}=1 I I$, Acta Arithmetica, (78) (4) (1997), 401-403.

[4] T. W. Cusick, The Diop. equat. $x^{4}-k x^{2} y^{2}+y^{4}=1$, Arch. Math., (59) (4) (1992), 345-347.

[5] C. Flaut, V. S. Shpakivskyi, An effic. meth. for solv. equat. in gen. quat. and octon. algeb., Advances Appl. Clifford Algebras, (25)(2) (2015), 337 350. 
[6] B. Gordon, T. S. Motzkin, On the zeros of poly. over div. ring, Transaction American Mathematical Soc., (116) (1965), 218-226.

[7] A. F. Horadam, Comp. Fib. numb. and Fib. quater., Amer. Math. Month., (70) (3) (1963), 289-291.

[8] A. İpek, On $(p, q)-F i b$. quat. and their Binet form., gener. func. and certain binom. sums, Advances Applied Cliff. Algebras, 27 (2) (2017), $1343-1351$.

[9] C. Kimberling, Fib. hyper., Fib. Quarterly, (28) (1) (1990), 22-27.

[10] W. L. McDaniel, Diop. repres. of Lucas seq., Fib. Quart., (33) (1995), 59-63.

[11] I. Niven, Equat. in quater., Amer. Math. Month., (48) (1941), 654-661.

[12] T. Sakkalis, K. Ko, G. Song, Roots of quat. poly.: theory and comput., Theoret. Computer Science, (800) (2019), 173-178.

[13] D.F. Scharler, J. Siegele, H.P. Schröcker, Quad. split quater. poly.: fact. and geom., Advances Appl. Cliff. Algebras, (30) (1)(2020), Art. 11, 23 pp.

[14] R. Serôdio, E. Pereira, J. Vitória, Comput. the zeros of quat. poly., Comput. Math. Appl., (42) (8-9) (2001), 1229-1237.

[15] V. S. Shpakivskyi, Lin. quat. equat. and their sys., Advances Appl. Cliff. Algeb., (21) (2011), 637-645.

[16] J. Zhigang, C. Xuehan, Z. Meixiang, A new method for roots of monic quat. quad. poly., Comput. Math. Appl.,(58) (9) (2009), 1852-1858.

Ilker AKKUS,

Department of Mathematics,

Kirikkale University,

71450, Kırıkkale, Turkey.

Email: iakkus.tr@gmail.com

Gonca KIZILASLAN

Department of Mathematics,

Kirikkale University,

71450, Kırıkkale, Turkey.

Email: goncakizilaslan@gmail.com 